\title{
Professionnalisation des directeurs associatifs par la formation continue en alternance
}

\author{
Le cas d'un réseau d'associations familiales \\ Professionalization of nonprofit organization managers \\ through continuing education. \\ The case of a group of family organizations
}

\section{Nadine Dubruc et Marylène Badour}

Numéro 322, octobre 2011

Le management dans l'ESS

Management in the social and solidarity Economy

URI : https://id.erudit.org/iderudit/1020722ar

DOI : https://doi.org/10.7202/1020722ar

Aller au sommaire du numéro

Éditeur(s)

Association Recma

ISSN

1626-1682 (imprimé)

2261-2599 (numérique)

Découvrir la revue

Citer cet article

Dubruc, N. \& Badour, M. (2011). Professionnalisation des directeurs associatifs par la formation continue en alternance : le cas d'un réseau d'associations familiales. Revue internationale de l'économie sociale, (322), 47-61.

https://doi.org/10.7202/1020722ar
Résumé de l'article

Cette communication cherche à apporter des éclairages sur la professionnalisation des structures de l'économie sociale et solidaire, à partir des enseignements d'un partenariat implicite mis en place avec un réseau d'associations familiales. Tout d'abord, nous verrons que la construction d'un dispositif de professionnalisation de dirigeants s'appuie sur la conjonction de cinq facteurs : la pression de l'obligation juridique, la prise en compte des besoins des futurs apprenants, le soutien d'un réseau national, la volonté des apprenants de se retrouver entre eux et, enfin, la réponse de l'organisme de formation, qui a su adapter un dispositif existant aux spécificités de cette promotion. Ensuite, nous avons pu montrer que la professionnalisation de dirigeants de structures de l'ESS à travers une formation repose également sur trois éléments du dispositif pédagogique : le groupe d'apprenants, le projet conduit dans le cadre de la formation et le mémoire professionnel réalisé pour valider une partie du diplôme. 


\section{PROFESSIONNALISATION DES DIRECTEURS ASSOCIATIFS PAR LA FORMATION CONTINUE EN ALTERNANCE} Le cas d'un réseau d'associations familiales

\footnotetext{
* Docteure en psychologie sociale, enseignant-chercheur, Ecole nationale supérieure des Mines, Saint-Etienne. Mél.: dubruc@emse.fr.

* Responsable du développement, Irup Saint-Etienne. Mél. mbadour@irup.com.

** Cet article est une version remaniée d'une communication présentée au colloque international "Gouvernance, management et performance des entreprises de I'ESS, quelles spécificités?" organisé à Lyon les 4 et 5 novembre 2010 par l'IAE de I'université de Jean-Moulin Lyon-3 et I'Esdes (Ecole supérieure de commerce et management de l'université catholique de Lyon). www.managementess2010-Iyon.fr.
}

par Nadine Dubruc* et Marylène Badour**

Cette communication cherche à apporter des éclairages sur la professionnalisation des structures de l'économie sociale et solidaire, à partir des enseignements d'un partenariat implicite mis en place avec un réseau d'associations familiales. Tout d'abord, nous verrons que la construction d'un dispositif de professionnalisation de dirigeants s'appuie sur la conjonction de cinq facteurs: la pression de l'obligation juridique, la prise en compte des besoins des futurs apprenants, le soutien d'un réseau national, la volonté des apprenants de se retrouver entre eux et, enfin, la réponse de l'organisme de formation, qui a su adapter un dispositif existant aux spécificités de cette promotion. Ensuite, nous avons pu montrer que la professionnalisation de dirigeants de structures de l'ESS à travers une formation repose également sur trois éléments du dispositif pédagogique: le groupe d'apprenants, le projet conduit dans le cadre de la formation et le mémoire professionnel réalisé pour valider une partie du diplôme $e^{* * *}$.
$\mathrm{D}$ ans cet article, nous souhaitons apporter un éclairage sur la professionnalisation des acteurs de l'économie sociale et solidaire (ESS) en nous focalisant notamment sur la professionnalisation des directeurs par un partenariat entre un réseau national et un institut de formation. Notre volonté est d'éclairer la professionnalisation dans l'ESS à trois niveaux, car "pour lire l'espace éducatif, il est commode de distinguer trois niveaux d'activité qui sont aussi trois niveaux d'analyse (macro, méso, micro)" (Leclercq, 2003, p. 502). Pour cela, nous avons choisi l'analyse d'un dispositif de formation de directeurs réalisé grâce à trois acteurs clés: une fédération nationale (appelée $\mathrm{ABC}$ dans cet article), un organisme de formation reconnu dans les formations de niveau 1 de dirigeant de structure associative et des directeurs d'association formés dans le cadre d'une promotion (encadré 1, en page suivante). Nous pensons apporter par ces trois niveaux une vision complémentaire de la professionnalisation, trop souvent cantonnée à l'analyse du vécu des apprenants et des outils de formation. 
Dans un premier temps, nous proposons une réflexion sur le concept de professionnalisation, pour dans un deuxième temps mettre en lien évolutions législatives et évolution de la formation des directeurs de structures sanitaires et sociales. Dans une troisième partie, nous verrons l'élaboration d'un dispositif de formation en appui sur un institut de formation de Saint-Etienne, l'institut régional universitaire polytechnique (Irup; www.irup.com), avant de repérer les éléments ayant contribué à la professionnalisation d'une promotion de directeurs.

\section{Professionnaliser des salariés en poste: un parti pris pédagogique}

Comme le soulignent Lemaitre (2011), Wittorski (2005) et Barbier (2006), la professionnalisation à travers les dispositifs de formation dans l'enseignement supérieur signifie une proposition d'un programme adapté aux conditions d'exercice d'une profession visée qui se situe dans des conceptions de formation. La question de la professionnalisation est portée avec une intention sociale depuis une dizaine d'années par les orientations nationales (ANI, 2003, loi de 2004) et européennes (conseil de Lisbonne, 2000...).

\section{Encadré 1}

\section{Trois niveaux de données}

Les résultats s'appuient sur une enquête qualitative et des données recueillies à partir d'entretiens semi-directifs de vingt et un directeurs d'association, de comptes rendus de réunions rédigés dans la durée de la conception du dispositif et de sa mise en place en 2008-2009, de textes de loi évoqués et de certains éléments des mémoires professionnels produits pour la validation du titre.

Nous avons ainsi trois niveaux de données, dont un premier, "macro ", qui porte plus particulièrement sur l'impact des lois sur l'évolution des organisations, notamment sur les exigences en termes de professionnalisation. Nous avons procédé à l'analyse des lois et décrets concernant le secteur des établissements sociaux et médico-sociaux et la formation des directeurs.

Un deuxième niveau, " méso ", reconstitue le système d'activité "professionnalisation d'un réseau à l'aide d'une formation " d'après une analyse de l'historique d'un partenariat entre le réseau $\mathrm{ABC}$ et l'institut de formation Irup. Les échanges de mails et les comptes rendus de réunions ont servi de matériau pour proposer une modélisation des étapes de construction d'un partenariat de professionnalisation.

Enfin, l'observation d'une promotion de directeurs $\mathrm{ABC}$ formés, et notamment l'analyse de leurs mémoires de fin de formation, permet de mettre en évidence, au niveau " micro ", la pertinence d'un dispositif de formation par alternance. Nous avons procédé à l'analyse des titres, des remerciements, des conclusions et des mots-clés de vingt mémoires professionnels produits par des directeurs pour la validation du titre « entrepreneur de l'économie sociale et solidaire ». 
«Professionnalisation» est un mot polysémique auquel nous pouvons raccrocher différents sens. Un premier sens renvoie selon la sociologie des professions à la notion de profession, considérée comme une organisation autonome qui se reconnaît par un statut, un discours propre, un corps propre de savoirs donnant au final une autorisation d'exercer. Une deuxième acception renvoie au but de former aux caractéristiques d'une profession par le développement des savoirs et des compétences. Dans ce sens, On associe souvent au mot " professionnalisation » les mots "métier » et "référentiel ». Enfin, un troisième sens a été largement diffusé dans les pratiques de formation en entreprise avec la notion de compétence à partir des années 70-80. Il s'agit moins de former à l'exercice d'une profession ou de développer les caractéristiques d'une profession, mais plus de développer la transversalité, la flexibilité, voire l'employabilité. Pour notre réflexion, nous souhaitons souligner certains aspects de la professionnalisation, qui serait alors: devenir un professionnel dans le cadre d'une formation co-construite en appui sur une analyse réflexive de sa pratique.

\section{Se professionnaliser: devenir un professionnel reconnu}

Certains auteurs parlent de la formation comme d'une construction identitaire, d'un nécessaire rapport à des dynamiques identitaires, des stratégies visant à dépasser certaines crises identitaires (Dubar, 2002). Ainsi, la vie est rythmée par un certain nombre de rites qui reconnaissent l'existence sociale de l'événement, qui assignent les individus dans les rôles respectifs (naissance, père, mère, communauté). Hugues (1996) insiste sur le rôle des rites de passage comme rites de transition dont l'intérêt est de permettre aux individus de passer d'une étape de leur existence à une autre. Le passage par une formation pour acquérir un diplôme reconnu par les pairs peut être considéré comme une façon de se faire reconnaître dans une profession. Dans le cas des formations sociales, les contraintes réglementaires ont fait évoluer notamment la reconnaissance des directeurs: d'un poste où l'on arrivait par ancienneté, par promotion interne, la règle devient l'obtention d'un diplôme bac +5 , devenu nécessaire. Nous verrons plus bas comment cela s'est opéré.

\section{Se professionnaliser par la formation réflexive}

Se professionnaliser, "pour un individu ou un groupe professionnel donné, c'est un ensemble de manières d'être, de penser et de faire qu'il s'approprie dans le cours même de son travail. Il sagit de propriétés sociales qui ont été construites dans le feu de l'action, dans l'épreuve des événements de la vie professionnelle (et en partie extraprofessionnelle, car on sait qu'il est difficile de séparer le temps de travail et le temps hors travail) (Demailly, 2001, p. 524). Schön (1994) caractérise le professionnel par sa capacité à apprendre dans et par la pratique. La connaissance se construit dans l'action, avec un processus d' " enquête " qui vise à donner du sens à la situation vécue grâce au contexte. La connaissance n'existe pas indépendamment des réalités 
du monde, elle se construit dans l'expérience et vise à étendre les capacités d'action. Schön appelle "discussion avec la situation " le processus par lequel le praticien crée du sens en interrogeant la situation par une série d'actions qui reçoivent une " réponse " en retour (si je fais ceci, il se passe cela). Il apparait donc nécessaire de porter un regard en formation sur l'expérience et plus particulièrement sur ce qui est discuté autour du métier. Une formation en alternance qui donne la place centrale à une expérience pour valider une partie du diplôme visant à professionnaliser s'appuie sur ce parti pris. Il s’agit ainsi de développer «une épistémologie du savoir caché dans l'agir professionnel ».

\section{Se professionnalisation par un dispositif de formation co-construit} "La professionnalisation "met en scène" des acquis personnels ou collectifs tels les savoirs, les connaissances, les capacités et les compétences. Bien plus, nous pourrions dire quelle réside dans le jeu de la construction etlou de l'acquisition de ces éléments qui permettront au final de dire de quelqu'un qu'il est un professionnel, c'est-à-dire qu'il est doté de la professionnalité (l'ensemble des connaissances, des savoirs, des capacités et des compétences caractérisant sa profession)" (Wittorski, 2005, p. 27). Professionnaliser des individus en formation consiste à proposer un dispositif de co-construction d'évolution individuelle.

Ces différents sens sont investis selon les acteurs. Les dispositifs de formation peuvent ainsi être regardés en fonction de ce qu'ils prescrivent et de ce qui est du réel, mais aussi en fonction de la façon dont se sont construites les formations (réponse à une obligation légale, concertation des acteurs...). Les étapes de la co-construction du dispositif entre le réseau, l'institut de formation et des représentants des directeurs seront décrites dans la troisième partie.

\section{Le mémoire, instrument clé de la professionnalisation}

Pour analyser le dispositif de professionnalisation des directeurs de l'ABC, nous nous sommes appuyées sur les théories socio-constructivistes, considérant la formation comme un processus de socialisation du sujet. Ainsi, la formation a pour objectif de permettre à un individu de s'inscrire dans un collectif, groupe social de professionnels, par la transmission des références et des valeurs de ce groupe d'appartenance. Progressivement, celui-ci permet à l'individu d'en devenir un sujet à part entière. Notre approche de la formation rejoint celle de Vygotski (2002) et «s'inscrit dans une conception sociale de la construction de la conscience qui considère que l'enseignant "prête" des formes de pensée, des instruments dont le destin, chez les apprenants, est de se transformer et de s'intégrer dans leur répertoire personnel de pensée et d'action " (Mayen, Mayeux, 2003, p. 20). Nous ne considérons pas le développement du sujet comme un processus interne et autonome, mais comme ayant un ancrage social fort.

Le développement de l'apprenant passe par les outils langagiers mis à sa disposition par le dispositif de formation ou mobilisés en situation 
de formation et notamment par l'intégration de ceux-ci. Lactivité «se professionnaliser » est donc constituée par le sujet actif, qui utilise des instruments (éléments à sa disposition) pour atteindre un mobile (objet visé par l'activité). Le mémoire professionnel donné à travailler durant le temps de formation est selon nous un instrument clé de la formation. Il serait un instrument du transfert entre le vécu du projet en tant que directeur et les approches théoriques vues en formation. Il permet de surmonter les contradictions perçues entre les différents systèmes d'activité dans lesquels travaille l'apprenant. Le dispositif de formation autour du projet, conduit par l'apprenant, impose de faire du lien entre la théorie et la pratique pour permettre le développement du futur « entrepreneur de l'économie sociale et solidaire ». Pour être surmontées, les contradictions doivent être rendues visibles. Elles entraînent alors un apprentissage qui oblige les sujets à modifier la structure de l'activité ou certains de ses composants (règles, objet, outils, organisation du travail). Dans ce cas, le mémoire permet l'apprentissage par expansion, car les différentes phases d'écriture et l'aboutissement du mémoire procèdent à une évolution de la perception du système par le sujet.

Nous porterons dans la dernière partie un regard privilégié sur une formation de dirigeants d'un réseau français d'associations sociales et leur professionnalisation par les outils proposés.

\section{La loi 2002-2: pression à la professionnalisation}

En 2004, un rapport du Conseil économique et social identifie les problèmes de recrutement dans la branche. "Pour le conseil, l'avenir du secteur sanitaire et social est intimement lié à celui de ses personnels. Afin de répondre dans les meilleures conditions possible aux enjeux sanitaires et sociaux, les solutions à mettre en cuvre passent, dès lors, par une dynamisation du recrutement, une réflexion sur le contenu comme sur les modalités des formations initiales et continues et, enfin, par une action continue de professionnalisation de l'ensemble des salariés du secteur " (Pinaud, 2004). Ce secteur se trouve en effet au cœur des problématiques de la génération papy-boom, avec un vieillissement des salariés et une prévision de départs à la retraite pour un quart d'entre eux. De plus, le secteur sanitaire et social bénéficie d'une insuffisance de personnel qualifié et d'un manque d'attractivité.

\section{Loi 2002-2 et exigences de professionnalisation des directeurs}

Selon la loi 2002-2, "les prestations délivrées par les établissements et services mentionnés aux $1^{\circ}$ à $12^{\circ}$ (de l'article 15$)$ sont réalisées par des équipes pluridisciplinaires qualifiées. Ces établissements et services sont dirigés par des professionnels dont le niveau de qualification est fixé par décret et après consultation de la branche professionnelle ou, à défaut, des fédérations ou organismes représentatifs des organismes gestionnaires d'établissements et 
(1) "Doit être titulaire d'une certification de niveau l enregistrée au Répertoire national des certifications professionnelles prévu à l'article L. 335-6 du Code de l'éducation le professionnel ayant reçu les délégations mentionnées aux troisième à septième alinéas de l'article D. 312-176-5 et qui, selon les situations: a) dirige ou administre l'un des groupements mentionnés à l'article L. 312-7, b) dirige un ou plusieurs établis sements ou services sociaux ou médico-sociaux répondant cumulativement, sur au moins trois exercices comptables clos consécutifs, au moins à deux des trois seuils fixés à l'article 44 du décret $n^{\circ}$ 2005-1677 du 28 décembre 2005 ; c) dirige le siège social, en application de l'article R. 314-87, d'un organismegestionnaire autorisé ») (art. D312-176-6 du Code de l'action sociale et des familles).

(2) «Les professionnels chargés de la direction d'un ou plusieurs établissements ou services sociaux ou médico-sociaux, en fonction à la date de publication du présent décret, qui ne justifient pas des qualifications prévues aux articles D. 312-176-6 à D. 312-176-8 et D. 312-176-10 du Code de l'action sociale et des familles, disposent pour obtenir ces qualifications d'un délai: de dix ans s'ils étaien en fonction à la date d'entrée en vigueur de la loi $n^{\circ} 2002-2 d u$ 2 janvier 2002; de sept ans s'ils ont été recrutés postérieurement. S'ils ne disposent pas de l'ancienneté de trois ans nécessaire pour s'engager dans un parcours de validation des acquis de l'expérience à la date de publication du présent décret, le délai de sept ans est augmenté de leur durée d'expérience manquante " (art. 2 du Code de I'action sociale et des familles). services sociaux et médico-sociaux concernés» (art. 15, L.312-1). Pour les structures concernées, la logique de la responsabilité est complétée et soutenue par une logique de la qualification.

La loi du 2 janvier 2002 fixe par décret le niveau de qualification des directeurs d'établissements sociaux et médico-sociaux, après consultation de la branche professionnelle ou des fédérations et organismes représentatifs des organismes gestionnaires d'établissements ou de services sociaux ou médico-sociaux. En 2005, la très grande diversité des conditions d'exercice et des profils des directeurs est constatée par la Direction générale des affaires sociales (DGAS). Après concertation, le décret $n^{\circ}$ 2007-221 du Code de l'action sociale et des familles et relatif aux modalités de délégation et au niveau de qualification des professionnels chargés de la direction d'un ou plusieurs établissements ou services sociaux ou médico-sociaux paraît le 19 février 2007. Il est ainsi précisé la nécessité d'obtenir un diplôme de niveau I ou II en fonction de la taille des établissements ${ }^{(1)}$. L'article suivant pondère le niveau I: "Sous réserve des dispositions législatives, réglementaires ou conventionnelles formulant des exigences supérieures, tout professionnel chargé de la direction d'un ou plusieurs établissements ou services sociaux ou médico-sociaux doit être titulaire d'une certification au moins de niveau II enregistrée au Répertoire national des certifications professionnelles prévu à l'article L. 335-6 du Code de l'éducation " (art. D312-176-7 du Code de l'action sociale et des familles). Quelles sont les conséquences de ces décrets sur la fonction de directeur?

\section{Professionnalisation des directeurs dans le social}

Dans le titre du décret, le mot «directeur " n'est pas utilisé: ce sont les "professionnels chargés de la direction d'un ou plusieurs établissements ou services sociaux ou médico-sociaux " qui sont mentionnés. Volonté de représenter la diversité des situations ou choix d'orienter le texte vers la fonction plutôt que vers la qualification? La capacité d'une personne à diriger une structure est évaluée en fonction du dipôme ou du titre acquis. A travers la volonté d'uniformiser le niveau de la fonction de direction de structure, nous observons un glissement vers la valorisation de la qualification plus que vers celle de la compétence. La loi oblige désormais à privilégier le recrutement de personnes à partir du diplôme au détriment du recrutement sur l'expérience.

Le décret tient compte des situations des directeurs en poste et, selon leur ancienneté dans la fonction, accorde un délai pour l'obtention d'un titre adéquat ${ }^{(2)}$. Ce délai peut permettre à certains directeurs en poste de terminer leur carrière jusqu'à la retraite. Retenons que la finalité de la professionnalisation "visée par les acteurs est la construction de règles protectrices régissant le rapport salarial d'une catégorie particulière de travailleurs " (Labruyère, 2000, p. 35) tout autant qu'une orientation sociale et éthique. A ces constats sur la professionnalisation dans les structures sanitaires et sociales, des réponses en termes de formation ont été apportées. 
La loi 2002-2 contraint les structures qui en relèvent à de nécessaires évolutions, voire révolutions, dans leurs organisations. Pour faire face, les directeurs de structure se sont parfois tournés vers la formation pour répondre aux contraintes, mais aussi avec la volonté de bénéficier des dispositifs de formation comme soutien à cette évolution. N'oublions pas, cependant, que "le travail social ne peut être qu'un produit de la discussion et de l'intersubjectivité. Tout intervenant a besoin de savoir ce qu'il fait, pourquoi il le fait, selon quels rapports entre son action et le système dans lequel il se trouve. Autrement, son travail se nourrirait de fantasmes et de tentations naïves de reconstruction du monde. Il y a aussi un autre piège à éviter, celui de la bureaucratisation du social" (Beaucourt, 2002, p. 13).

C'est à travers l'exemple d'un partenariat entre un réseau de structures, des directeurs et un organisme de formation que nous allons tenter de le vérifier. Nous sommes là dans ce que nous qualifierons de "forme de dispositifs de formation partenariaux plus complexes, multi-acteurs, nécessitant la négociation d'un enjeu collectif entre l'entreprise et l'organisme de formation »(Sorel, Wittorski, 2005, p. 19).

\section{La construction d'un partenariat avec un organisme de formation}

Le point de départ du partenariat, objet du présent article, a été le contact pris en 2008 par un groupe de représentants de directeurs d'associations du réseau $\mathrm{ABC}$ du secteur social et médico-social avec l'Irup. Le partenariat se concrétise en janvier 2009 par le démarrage d'une session de formation dédiée aux directeurs de ce réseau. Pour parvenir à ce résultat, trois étapes ont été nécessaires: l'exposé des besoins; la mobilisation de la structure de formation du réseau; l'adaptation du programme de formation et des modalités pédagogiques aux besoins spécifiques des directeurs.

\section{Un réseau et des associations militantes pour la famille}

Le réseau $\mathrm{ABC}$ observé est composé d'une union nationale et d'associations départementales, instituées par une ordonnance après la Seconde Guerre mondiale. La légitimité de ce réseau a été portée par l'Etat et ces associations sont nées d'une volonté politique et des besoins de cette époque post-guerre d'écoute et de prise en compte des familles. La gouvernance de ces associations est aussi particulière. Dans les conseils d'administration siègent des représentants des associations familiales: Association familles rurales (AFR), ADMR, Associations familiales laïques (Cnafal), Familles de France... Chaque association départementale est sous la responsabilité d'un président qui travaille lui-même avec un directeur salarié. Ces associations forment donc un réseau de production de services (tutelle, aide à la parentalité, action éducative en milieu ouvert, services familiaux...) et une institution de représentation légale des familles auprès de l'Etat. Chaque directeur porte ces valeurs de défense de la famille de façon très forte auprès du réseau national. 
Un certain militantisme se ressent dans les discours et les pratiques des dirigeants de ces structures.

Nous considérons les associations du réseau $\mathrm{ABC}$ comme faisant partie de l'ESS. Les associations étudiées respectent en effet les critères de liberté d'adhésion, de non-lucrativité, de gestion démocratique et d'utilité sociale du projet.

\section{L'Irup: la formation à partir de l'expérience professionnelle}

La culture d'action éducative portée par l'Irup, établissement d'enseignement supérieur en alternance au statut associatif, créé en 1995 à Saint-Etienne, est celle de la professionnalisation. Il faut retenir par professionnalisation "un processus finalisé de transformation de compétences en rapport avec un processus de transformation d'activités " (Sorel, Wittorski, 2005, p. 126). Le cœur de métier de l'institut est l'accompagnement des apprenants en situation professionnelle.

Toutes les formations de l'Irup sont professionnalisantes et diplômantes. Ce sont des formations supérieures qui aboutissent à la délivrance de diplômes ou de certifications professionnelles $(\mathrm{bac}+2$ à bac +5$)$ inscrites au Répertoire national des certifications professionnelles (RNCP). Les formations s'inscrivent dans une démarche pédagogique qui associe étroitement mise en situation professionnelle et formation académique. Elles répondent à une double logique: acquisition de compétences et évolution identitaire pour la personne en formation, et dynamique de progrès pour l'entreprise. Lapprenant est encadré par un double tutorat: un tuteur Irup et un tuteur entreprise.

L'Irup forme en 2011 près de cinq cents apprenants par an (techniciens supérieurs, cadres intermédiaires, cadres dirigeants...). Son pôle management, qui nous intéresse ici, propose des formations dans les domaines de la comptabilité-finance, de la gestion appliquée à l'économie sociale, de la stratégie-organisation-ressources humaines.

Culturellement attaché aux valeurs associatives et soucieux d'apporter une réponse formative à la problématique de professionnalisation des acteurs des organisations de l'économie sociale, l'Irup a co-construit un parcours de formation adapté aux besoins des cadres dirigeants des entreprises associatives dès 2006. Ce cursus répond aux critères de professionnalisation en plaçant en son centre la situation professionnelle des apprenants avec l'objectif de l'« exploiter » à des fins formatives.

\section{Exposé des besoins}

Lors d'une première rencontre à Saint-Etienne, le groupe de représentants des directeurs expose la problématique de professionnalisation des cadres dirigeants de son réseau national associée à la nouvelle exigence en matière de qualification des directeurs d'établissements ou services du secteur social et médico-social (décret 2007-221 du 19 février 2007). Les directeurs expriment le fait que leurs associations subissent "un processus d'isomorphisme mimétique lié à l'incertitude et à l'ouverture à la 
concurrence. Elles tendent en effet à rechercher des modèles sur lesquels se référer et à adopter des solutions déjà connues et reconnues comme efficaces" (Marival, 2008, p. 8).

\section{Mobilisation de la structure de formation du réseau}

Une fois l'expression des besoins collectée, la structure de formation du réseau, dont la mission consiste à coordonner la formation sur le plan départemental et régional et à s'ouvrir vers les organismes associatifs ou institutionnels à vocation sociale, s'est mise en relation avec l'institut de formation. Le cadre conventionnel du partenariat a été discuté et les parties prenantes ont fait le choix de l'informel, prenant ainsi le parti de privilégier la relation de confiance qui s'était nouée. Ce choix illustre également une certaine liberté d'action des associations du réseau en matière de formation. Entre les deux opérateurs de formation, une collaboration de qualité s'est rapidement installée, mettant en évidence une grande capacité de mutualisation, de coopération et des relations interpersonnelles déterminantes.

Les premières discussions engagées à la fin de l'été 2008 ont abouti en octobre 2008, preuve s'il en est que, lorsque l'enjeu est fort et que les objectifs sont explicités, la construction de la réponse formative peut être rapide. Nous pouvons reprendre la qualification de Sorel et Wittorski (2005) d'une demande d'élaboration d'un dispositif de formation nouveau inscrit dans un contexte spécifique. Cette demande correspondrait à une volonté d'introduction de changement des pratiques de travail en vue de rétablir les structures et de les mettre en conformité avec leur environnement.

\section{Une formation adaptée aux besoins spécifiques des directeurs}

Ladhésion des directeurs au parcours de formation proposé tient au fait qu'ils sont à l'origine de l'expression du besoin et de la présélection de l'institut de formation. Ensuite, ils ont été associés, sous différentes formes, à l'étape d'adaptation du programme de formation et de définition des modalités pédagogiques, rendant ainsi compatible leur activité professionnelle avec leur investissement dans la formation.

Le choix du lieu de formation s'est porté sur les locaux du siège social du réseau à Paris, rendant ainsi lisible l'engagement de celui-ci dans le partenariat et facilitant les déplacements des directeurs concernés. Le rythme d'alternance de la formation retenu a permis le maintien dans des conditions quasi normales de leur activité professionnelle. L'institut de formation a fait preuve de souplesse et d'innovation dans la mise au point du parcours (alternance, modalités pédagogiques variées...).

Autrement dit, les partenaires ont pris un risque qu'ils ont complètement assumé: risque pour le réseau d'associations de choisir un institut de formation peu connu, qui a démontré son professionnalisme; risque de choisir une formation hors du champ universitaire, hors du champ social et médico-social; risque pour l'institut de formation d'organiser 
une formation en alternance hors site, notamment à l'aide d'outils pédagogiques à distance.

Ces trois étapes sont fondatrices de ce partenariat entre un réseau d'associations du secteur social et médico-social et un institut de formation. Elles mettent en avant le positionnement original du réseau national, les qualités d'adaptation et le professionnalisme des opérateurs de formation. C'est en appui sur ce dispositif co-construit que va pouvoir se dérouler la professionnalisation d'une première promotion.

\section{Une formation de directeurs en alternance}

L'intérêt des formations par alternance est de permettre des allersretours réguliers entre la théorie et la pratique, entre le monde des savoirs et celui de l'entreprise. Pour illustrer la façon dont un dispositif de suivi dans une formation participe à la professionnalisation de directeurs, nous prenons l'exemple de la formation de vingt et un apprenants, directeurs ou directeurs adjoints à l'ABC, formés dans le cadre de la certification «entrepreneur de l'économie sociale et solidaire ". Cette formation ouverte aux salariés en formation continue a en effet pour objectif de permettre aux apprenants de valider en douze mois des compétences de dirigeant de l'ESS. La validation de la certification s'appuie sur une double évaluation: d'abord celle de savoirs théoriques, puis une validation des compétences professionnelles, exprimées dans le cadre de la conduite d'un projet de développement stratégique dans une structure de l'ESS. Les vingt et un dirigeants ayant suivi la formation se répartissent sur tout le territoire français. Il s'agit de:

- vingt directeurs, avec 2 à 525 salariés encadrés, pour une moyenne de la promo de 100 salariés encadrés, et un adjoint de direction;

- quatre femmes et dix-sept hommes, de 50 ans en moyenne;

- trois apprenants à bac +2 ; huit apprenants à bac +3 ; neuf apprenants à bac +4 ; un apprenant à bac +5 .

Sur la promotion, un seul n'a pas finalisé son mémoire et n'a donc pas validé la formation.

\section{Remise en cause du genre professionnel}

$\mathrm{Du}$ recrutement à la soutenance finale, un ensemble de supports, de moments permettent à l'individu de se positionner ou de tenter de le faire tout au long de la formation. Nous avions montré précédemment (Dubruc, 2009) que se professionnaliser vers un métier est un processus qui se réalise par:

- des réflexions individuelles, des temps de prise de recul sur le métier visé et donc sur son orientation;

- des échanges dans un groupe d'apprenants;

- une confrontation à la réalité d'un contexte, d'un métier ; 
- des échanges avec l'équipe pédagogique, les intervenants professionnels du secteur.

Ces différents éléments sont autant de temps de construction et de réflexion sur les représentations du métier.

Dans le cas des directeurs observés, les évolutions du secteur peuvent être considérées comme une remise en cause du genre professionnel (Clot, Faïta, 2000). Le genre professionnel permet de donner à la situation et à l'activité de direction une signification. Il s'est agi pour ce groupe de professionnels de retrouver un genre au travers des conventions tacites du métier qui donnent des repères pour l'action. Se retrouver en formation, c'est se donner la possibilité de discuter des éléments vus en cours, d'échanger des points de vue sur la place du marketing, des choix de gestion à réaliser, des orientations des politiques gouvernementales, régionales et locales... Créer ou consolider son réseau de directeurs de mêmes associations, échanger sur des réalités proches ou au contraire très différentes sont autant d'éléments clés qui sont abordés dans les entretiens et semblent avoir aidé à la professionnalisation et à la construction d'un genre partagé " directeur d'ABC ». La notion de réseaux confortés se retrouve notamment dans les remerciements de deux groupes de quatre personnes qui se nomment clairement, qui se remercient pour la " cordialité des relations " ou parce qu' "ensemble ils ont progressé dans leurs travaux " et ont eu des "échanges fructueux", ce qui a fait de cette année "une année de formation et d'amitié "... Ainsi, "l'expérience se discute et s'explique, se réfléchit, elle est l'objet d'interactions dans lesquelles elle se construit et prend forme, s'amarre éventuellement aux fictions et aux mythes propres au groupe professionnel ou à ses routines " (Demailly, 2001, p. 525).

\section{Projets et logique de professionnalisation}

Les projets conduits pendant la formation pour valider le titre visé servent les objectifs stratégiques des directeurs observés. Ainsi, si nous prenons les sujets de mémoire, synthèse des projets conduits pendant la formation, nous constatons qu'ils correspondent aux préoccupations des structures en tant qu'adaptation aux exigences juridiques de la loi 2002-2.

A partir de la typologie de Marival (2008) des stratégies de légitimation possibles pour faire face à l'environnement, nous classons les projets conduits dans le tableau 1 (en page suivante).

Une partie des projets (neuf sur dix-neuf) concerne le positionnement de l'association dans son environnement, inscrivant l'association dans la conformité des attentes des acteurs publics. On aura là par exemple des projets de "mise en auvre de démarche qualité et d'applicabilité de la loi 2002-2", d'" adaptation et [de] modernisation des services", de "mise en conformité »... Les lois du 2 janvier 2002-2 et de 2007 imposent aux établissements de garantir une évaluation interne et externe régulière. Des procédures de contrôle sont mises en place sur les investissements et les coûts, la qualité, les prix. "En découvrant "la 2002-2" et les logiques de la qualité qu'elle contient, de nombreux professionnels perçoivent différemment 


\section{Tableau 1 \\ Les stratégies de légitimation dans les projets des apprenants}

\begin{tabular}{|l|l|}
\hline Processus & $\begin{array}{l}\text { Nombre de mémoires } \\
\text { correspondants (sur 19) }\end{array}$ \\
\hline 1. Stratégie de conformité à travers des processus d'isomorphisme institutionnel & 9 \\
\hline 2. Stratégie de développement et de croissance & 5 \\
\hline 3. Stratégie d'expertise & 0 \\
\hline 4. Stratégie de coalition d'acteurs & 5 \\
\hline
\end{tabular}

leur métier, leur mission et leur légitimité. L'envie de défendre leurs valeurs succède au premier effroi, le plaisir et la fierté de les savoir reconnues les encouragent à intégrer le cercle vertueux du management de la qualité par la 2002-2 " (Seintein, 2004, p. 72). Savignat (2009) reconnaît que la loi 2002-2 vue sous l'angle "logique qualité " peut constituer un apprentissage évaluatif, faisant prendre conscience aux acteurs que "la qualité est un construit et non un donné ». Il reproche cependant aux démarches qualité leur caractère normatif se résumant trop souvent à la mesure d'un écart entre le prescrit et le réalisé, ainsi que leur orientation techniciste dominée par la méthodologie. Etre conforme renseigne sur l'effectivité et non sur la pertinence de la pratique.

Les projets autour de stratégies plus volontaristes, plus " actives ", avec souvent la volonté de limiter la dépendance à l'environnement, sont plus nombreux si nous regroupons les stratégies de développement et les stratégies de coalition. Pour cinq projets, il s'agit de créer de nouveaux services et pour cinq autres de développer des coalitions d'acteurs pour partager le pouvoir, coordonner des interdépendances avec la mise en place de coopérations départementales, voire régionales. Les associations se voient dans l'obligation de passer d'une logique de besoins à une logique de moyens et de gestion d'enveloppes allouées avec les dotations globales de financement. Marival (2008) constate alors la promotion de nouveaux outils de coopération tels que les groupements de coopération sociale ou médico-sociale (GCSMS) ou l'incitation à atteindre des tailles critiques, ce qui correspond à la majorité des projets choisis par les directeurs en formation (dix sur dix-neuf).

A la logique de maintien d'activité des dirigeants de structure observés et d'adaptation aux nouveaux modes de financement, nous aurions pu analyser plus finement les stratégies de financement adoptées par les dirigeants selon trois types:

- une stratégie de maintien du niveau de financement;

- une stratégie de développement de financements à travers la mise en place de nouvelles activités; 
- une stratégie de financement par agrégation inter-structures sur un territoire.

Donner la possibilité, dans la formation, de se former autour de la situation professionnelle permet à des directeurs en poste de mettre au travail leur réalité professionnelle et de s'en saisir à travers les éléments de la formation. Malgré les réticences du départ et les discussions sur l'intérêt de prendre un projet au cœur de leurs préoccupations, nous retrouvons des éléments sur la professionnalisation au travers des conclusions.

Sur douze conclusions analysées, dix mettent en évidence en quoi le projet conduit a permis à l'apprenant de participer à la «transformation du secteur " en lien avec " la contrainte de diminution des fonds publics " (citée huit fois). De même, la conduite du projet est liée pour neuf d'entre eux à une évolution personnelle qui a permis par exemple à l'un d'eux de "clarifier [son] positionnement et [sa] mission dans la fonction de direction", pour un autre de "constater les progrès accomplis " ou encore "d'être riche d'enseignement [...] permettant de se re-questionner, de se positionner et de prendre de la hauteur et ce recul qui [leur] est tant nécessaire".

\section{Conclusion}

La professionnalisation d'un directeur de structure de l'ESS s'entend comme un dispositif qui n’a pas réduit ce directeur à "un rôle de "consommateur" du savoir qui lui est proposé [...]. Au contraire, il lui revient de le produire en formation, sous la conduite du formateur, en vue de sa mise en cuvre en situation de travail: il est alors auteur des savoirs - ou co-auteur de ces derniers autant qu'il en est l'apprentisseur" " (Sorel, Wittorski, 2005, p. 251)

Nous avons pu ainsi montrer que, pour les apprenants observés, il a fallu réussir à faire coïncider des enjeux qui pourraient paraitre contradictoires: se former pour répondre à une obligation légale, garder son identité de directeur tout en acceptant de se former et accepter le risque de se voir confronté à un niveau de formation, concilier responsabilités et absences pour une formation...

Comme éléments clés de la construction de ce dispositif de professionnalisation, nous identifions la pression de l'obligation juridique, la prise en compte des futurs apprenants, de leurs besoins, le soutien de ces apprenants par le réseau national, la volonté de ceux-ci de se retrouver entre eux, la réponse de l'organisme de formation, qui a su adapter un dispositif existant aux spécificités de cette promotion.

Comme instruments clés de la professionnalisation, nous mettons en avant trois éléments du dispositif pédagogique: le groupe d'apprenants, le projet conduit dans le cadre de la formation et le mémoire professionnel réalisé pour valider une partie du diplôme.

A titre de comparaison, l'institut de formation a développé dans le même temps un partenariat avec un réseau national d'associations du secteur des 
services à la personne. Dans la construction du partenariat, la rencontre s'est faite avant tout avec le réseau national, et la proposition pédagogique apportée a privilégié la prise en compte de l'expérience avec une orientation vers un dispositif de validation des acquis de l'expérience (VAE). Il faudrait aller plus loin dans une analyse plus méthodologique de ce dispositif, mais a priori il semblerait que ceux qui sont allés jusquau bout ont mobilisé deux des trois instruments cités plus haut: le " groupe » créé de postulants à la VAE et le dossier de VAE (qui n'est pas un mémoire professionnel).

Dans le cas de ce réseau français d'associations familiales, 2008 était une année importante pour préparer l'obligation de formation de ses directeurs départementaux. Le poids du réseau national et sa façon de mobiliser ses associations adhérentes sont deux éléments importants dans la réussite d'un tel dispositif. C'est ainsi que la mobilisation des directeurs, décisionnaires principaux dans ce type d'organisation, a ensuite été primordiale lors de la mise en place de formation pour les encadrants de niveau intermédiaire sur une formation de niveau II. 


\section{Bibliographie}

Ardoino J., 2000, De l' accompagnement", en tant que paradigme à René Lourau, Pratiques de formation-Analyses, université Paris-8, formation permanente.

Barbier J.-M., 2006, « Les nouvelles voies de la professionnalisation ", in Lenoir Y., BouillierOudot M.-H. (dir.), Savoirs professionnels et curriculum de formation, Presse de l'université de Laval (Canada), p. 67-81.

Beaucourt C., 2002, "La gestion des ressources humaines dans le secteur social ", Les Cahiers de la recherche, Claree.

Clot Y., Faïta D., 2000, "Genres et styles en analyse du travail ", Travailler, 4, p. 7-42. Demailly L., 2001, " La rationalisation du traitement social de l'expérience professionnelle ", Revue des sciences de l'éducation, 27(3), p. 523-542.

Dubar C., 2002, La socialisation: construction des identités sociales et professionnelles, Paris, A. Colin, $3^{\mathrm{e}}$ édition.

Dubruc N., 2009, "Le stage en entreprise: facteur de développement? Un dispositif de formation structuré par des instruments langagiers. Rôle du stage en formation initiale d'ingénieurs ", thèse de doctorat en psychologie sociale, université Lumière Lyon-2.

Hugues E. C., 1996, Le regard sociologique, Paris, EHESS.

Labruyere C., 2000, "Professionnalisation: de quoi parlent les chercheurs, que cherchent les acteurs? ", Formation emploi, n ${ }^{\circ} 70$, p. 31-42.

Leclercq G., 2003, «Interpréter et développer des dispositifs de formation ", Revue des sciences de l'éducation, vol. 29, n 3, p. 499-524.
Lemaître D., 2011, « Enjeux idéologiques de la professionnalisation dans l'enseignement supérieur ", in Actes du colloque "Les courants de la professionnalisation", Angers, 8-10 juin.

Marival C., 2008, "Quels effets de l'action publique sur les formes d'entrepreneuriat dans l'ESS ? Le cas des associations du secteur social et médico-social », colloque «Economie sociale et solidaire: nouvelles pratiques et dynamiques territoriales ", Institut d'économie et de management de Nantes-IAE, Nantes, 29-30 septembre 2008, 20 p.

Mayen P., Mayeux C., 2003, « Expérience et formation ", Revue Savoirs, p. 15-44.

Pinaud M., 2004, « Le recrutement, la formation et la professionnalisation des salaries du secteur sanitaire et social ", Notes d'Iéna, $\mathrm{n}^{\circ} 183,2$ juillet.

Savignat P., 2009, Evaluer les établissements et les services sociaux et médico-sociaux, Dunod, coll. "Action sociale".

Schön D., 1994, Le praticien réflexif: à la recherche du savoir caché dans l'agir professionnel, Les Editions logiques.

Sentien D., 2004, « La loi « 2002-2 : du management de la rupture au management par la démarche d'amélioration continue ", Empan, 3 (no 55), p. 62-72.

Sorel M., Wittorski R. (dir.), 2005, La professionnalisation en actes et en questions, L'Harmattan, coll. "Action et savoir".

Vygotski L., 2002, Pensée et langage, Paris, La Dispute (1 ${ }^{\text {re }}$ édition 1934).

Wittorski R. (dir.), 2005, Formation, travail et professionnalisation, L'Harmattan, coll. "Action et savoir». 